\title{
MRI Patterns of Extrapontine Lesion Extension in Diffuse Intrinsic Pontine Gliomas
}

\author{
(D) L. Makepeace, (D) M. Scoggins, (D) B. Mitrea, (D). Li, (D)A. Edwards, (D) C.L. Tinkle, (D) S. Hwang, (D) A. Gajjar, and (D) Z. Patay
}

\begin{abstract}
BACKGROUND AND PURPOSE: Diffuse intrinsic pontine glioma is a devastating childhood cancer that despite being primarily diagnosed by MR imaging alone, lacks robust prognostic imaging features. This study investigated patterns and quantification of extrapontine lesion extensions as potential prognostic imaging biomarkers for survival in children with newly diagnosed diffuse intrinsic pontine glioma.
\end{abstract}

MATERIALS AND METHODS: Volumetric analysis of baseline MR imaging studies was completed in 131 patients with radiographically defined typical diffuse intrinsic pontine gliomas. Extrapontine tumor extension was classified according to the direction of extension: midbrain, medulla oblongata, and right and left middle cerebellar peduncles; various extrapontine lesion extension patterns were evaluated. The Kaplan-Meier method was used to estimate survival differences; linear regression was used to evaluate clinical-radiographic variables prognostic of survival.

RESULTS: At least 1 extrapontine lesion extension was observed in 125 patients (95.4\%). Of the 11 different extrapontine lesion extension patterns encountered in our cohort, 2 were statistically significant predictors of survival. Any extension into the middle cerebellar peduncles was prognostic of shorter overall survival $(P=.01)$, but extension into both the midbrain and medulla oblongata but without extension into either middle cerebellar peduncle was prognostic of longer overall survival compared with those having no extension $(P=.04)$ or those having any other pattern of extension $(P<.001)$.

CONCLUSIONS: Within this large cohort of patients with typical diffuse intrinsic pontine gliomas, 2 specific extrapontine lesion extension patterns were associated with a significant overall survival advantage or disadvantage. Our findings may be valuable for risk stratification and radiation therapy planning in future clinical trials.

ABBREVIATIONS: DIPG = diffuse intrinsic pontine glioma; EPLE = extrapontine lesion extension; $\mathrm{ETV}=$ extrapontine tumor volume; $\mathrm{MCP}=$ middle cerebellar peduncle; OS = overall survival; PFS = progression-free survival; PTV = pontine tumor volume; RT = radiation therapy; TTV = total tumor volume

$\mathrm{H}_{\mathrm{t}}$ igh-grade gliomas comprise $10 \%-15 \%$ of pediatric brain tumors and cause half of the deaths occurring as a result of CNS neoplasms in children. ${ }^{1,2}$ Of pediatric high-grade gliomas, those occurring in the pons, formerly referred to as diffuse intrinsic pontine glioma (DIPG), are most common and have the worst prognosis, with a 2 -year survival rate of $<10 \%$. ${ }^{2,3}$

Received November 1, 2018; accepted after revision December 10, 2019.

From the Departments of Diagnostic Imaging (L.M., M.S., B.M., A.E., S.H., Z.P.), Biostatistics (Y.L.), Radiation Oncology (C.L.T.), and Oncology (A.G.), St. Jude Children's Research Hospital, Memphis, Tennessee.

This work was supported by the National Institutes of Health Cancer Center Support Grant P30 CA21765, the National Cancer Institute grant R25CA23944, and American Lebanese Syrian Associated Charities (ALSAC).

Please address correspondence to Zoltan Patay, MD, PhD, Department of Diagnostic Imaging, St. Jude Children's Research Hospital, 262 Danny Thomas Pl, MS220, Memphis, TN 38105-3678; e-mail: Zoltan.Patay@STJUDE.ORG

- Indicates open access to non-subscribers at www.ajnr.org

http://dx.doi.org/10.3174/ajnr.A6391
DIPGs present with characteristic MR imaging features, ${ }^{4}$ explaining the high accuracy of the initial diagnostic imaging work-up $(>95 \%)^{5,6}$ and the low rates of biopsies required before treatment. On MR imaging, the typical DIPG appears as an expansile, dominantly T2-hyperintense, usually inhomogeneous, intra-axial brain stem mass involving most of the pons but originating from the ventral pons (or basis pontis). ${ }^{1}$ These tumors often show some signal enhancement after IV injection of gadolinium-based contrast agents and ventral exophytic growth, leading to engulfing of the basilar artery. ${ }^{7}$ Extrapontine lesion extensions (EPLEs) are also common in DIPG.

Although outcomes in DIPG remain grim overall, differences in disease course across patients are also well-known. Therefore, the need for imaging features that can inform noninvasive biologic characterization, aid early prognostication, and guide the development of risk-stratification schemes for future clinical trials persists. Hence, the quest for robust conventional (or 
advanced) prognostic imaging biomarkers of DIPG continues in the pediatric neuroimaging and neuro-oncology communities.

In 2008, Hargrave et $\mathrm{al}^{5}$ studied several conventional MR imaging features, including local extension, exophytism, percentage of pontine volume affected, T1 or T2 signal heterogeneity, metastasis, tumor dimension, and margin definition and found none to be predictive of survival. Jackson et $\mathrm{al}^{6}$ have also attempted to describe imaging characteristics of long-term survivors of DIPG, but the value of the findings was somewhat limited by the small number of long-term survivors. In 2015, Jansen et $\mathrm{al}^{8}$ suggested that the duration of clinical symptoms before diagnosis, age at diagnosis, and ring enhancement within the tumor lesion in postcontrast T1weighted images may have prognostic value in DIPG. Others have suggested that ADC or ADC histogram metrics derived from diffusion-weighted MR imaging may identify prognostically distinct subgroups and correlate with survival in patients with DIPG. ${ }^{9,10}$ Most recently in 2018, Hoffman et $\mathrm{al}^{2}$ assessed clinical-radiographic characteristics and their relationship with outcomes in a collaborative study that included $>1000$ patients with DIPG. In the largest population of DIPGs ever studied, they identified increased craniocaudal tumor dimension and higher rates of EPLE, tumor necrosis, and ring enhancement at diagnosis as significantly associated with shorter overall survival (OS) on univariate analysis, but only ring enhancement was significantly associated with OS on multivariate analysis. This outcome contrasts with the findings of Poussaint et $\mathrm{al}^{11}$ in which larger tumor volumes were associated with longer survival (in that study, EPLEs were not analyzed separately but were presumably included), though enhancement at diagnosis was associated with shorter survival in their cohort as well.

Although prior studies, including the last 2 references cited above, suggest that EPLE may be relevant to and explain some of the differences in the clinical course and OS in patients with DIPG, methodologic differences hamper the unambiguous interpretation of these findings. Therefore, we sought to revisit this matter and clarify, in more specific terms, the potential prognostic value of EPLE at diagnosis in DIPG. To that end, we conducted a retrospective study using systematic and standardized analysis of imaging data focusing on the descriptive (ie, patterns) and quantitative (ie, overall burden) evaluation of EPLE and correlated those factors with key survival metrics in the largest, single-center cohort of patients with radiographically confirmed typical DIPG to date.

\section{MATERIALS AND METHODS Study Population}

We identified all patients (younger than 18 years of age) with newly diagnosed DIPG who were evaluated at our institution at the time of diagnosis during the 17-year period between January 1, 2000, and December 31, 2017 (193 patients). Patients who had been previously treated or had metastatic disease at diagnosis were not included. For the purposes of this review, we requested approval from our institutional review board to retrospectively evaluate the clinical and imaging data collected during the treatment and follow-up of patients. This request, in conjunction with a waiver of repeat consenting when the patient had been enrolled in a clinical trial, was granted.

Patients who did not have an adequate baseline MR imaging examination suitable for segmentation and coregistration were excluded $(n=36)$. Additionally, patients who were alive on May 1,2018 , were excluded because final survival data could not be collected for them $(n=24)$. Two of the remaining 133 patients were excluded because in retrospect, they were determined to have non-DIPG tumors of the brain stem (ie, not fulfilling the imaging criteria of the typical DIPG). One patient exhibited supratentorial tumor spread diffusely into the cerebral hemispheres with a gliomatosis cerebri growth pattern and a mixed glial/neuronal histopathologic phenotype, and 1 patient underwent surgical resection, which revealed a high-grade glioma of the medulla oblongata. Overall, 131 patients were included in our retrospective chart review and diagnostic MR imaging review study; 108 patients were enrolled in a total of 8 different therapeutic clinical trials, and 23 patients were treated on individualized treatment plans. Details of the 3 clinical trials accounting for the largest patient accrual sources, including treatment protocols and outcomes, have been reported previously. ${ }^{12-14}$

\section{Clinical Variables}

Clinical information was extracted from the patients' medical records, including demographics, age at diagnosis, progressionfree survival (PFS), and OS. Age at diagnosis was defined as the time from the date of birth to the diagnostic MR imaging study performed at our institution; PFS was defined as the time from diagnosis to disease progression as determined by a combination of clinical and radiologic parameters, with minor differences across treatment protocols. Typically, progressive disease was defined by any of the following: 1) neurologic abnormalities or worsening neurologic status not explained by causes unrelated to tumor progression (eg, seizures, anticonvulsant toxicity, electrolyte disturbances, sepsis); 2) a $>25 \%$ increase in the product of the maximum perpendicular diameters of the tumor lesion by imaging; 3) the presence of new metastatic disease; or 4) increasing doses of dexamethasone required to maintain a stable neurologic status. OS was defined as the time from diagnosis to death from any cause. The presence or absence of metastasis at the time of progression was recorded as well as the distribution pattern of metastasis: intracranial, intraspinal, or both.

\section{Image Processing}

Baseline MR imaging studies of the brain (ie, first diagnostic MR imaging examinations at our institution) were obtained for all patients within 2 weeks before initiation of treatment. After the baseline MR imaging study, patients either consented to participate in a therapeutic clinical trial or elected an individualized, nonprotocol treatment plan. Most studies $(n=114)$ were performed on $3 \mathrm{~T}$ magnets (the remaining on $1.5 \mathrm{~T}$ ) and under various forms of anesthesia. Standard brain MR imaging was performed in all patients, including axial T1WI, postcontrast axial T1WI, and postcontrast 3D-T1WI. Postcontrast axial FLAIR imaging was also available for all except 4 patients for whom sagittal 3D-FLAIR imaging was used for segmentation. For 1 patient, who had no axial T2WI, sagittal 3D-T2WI 

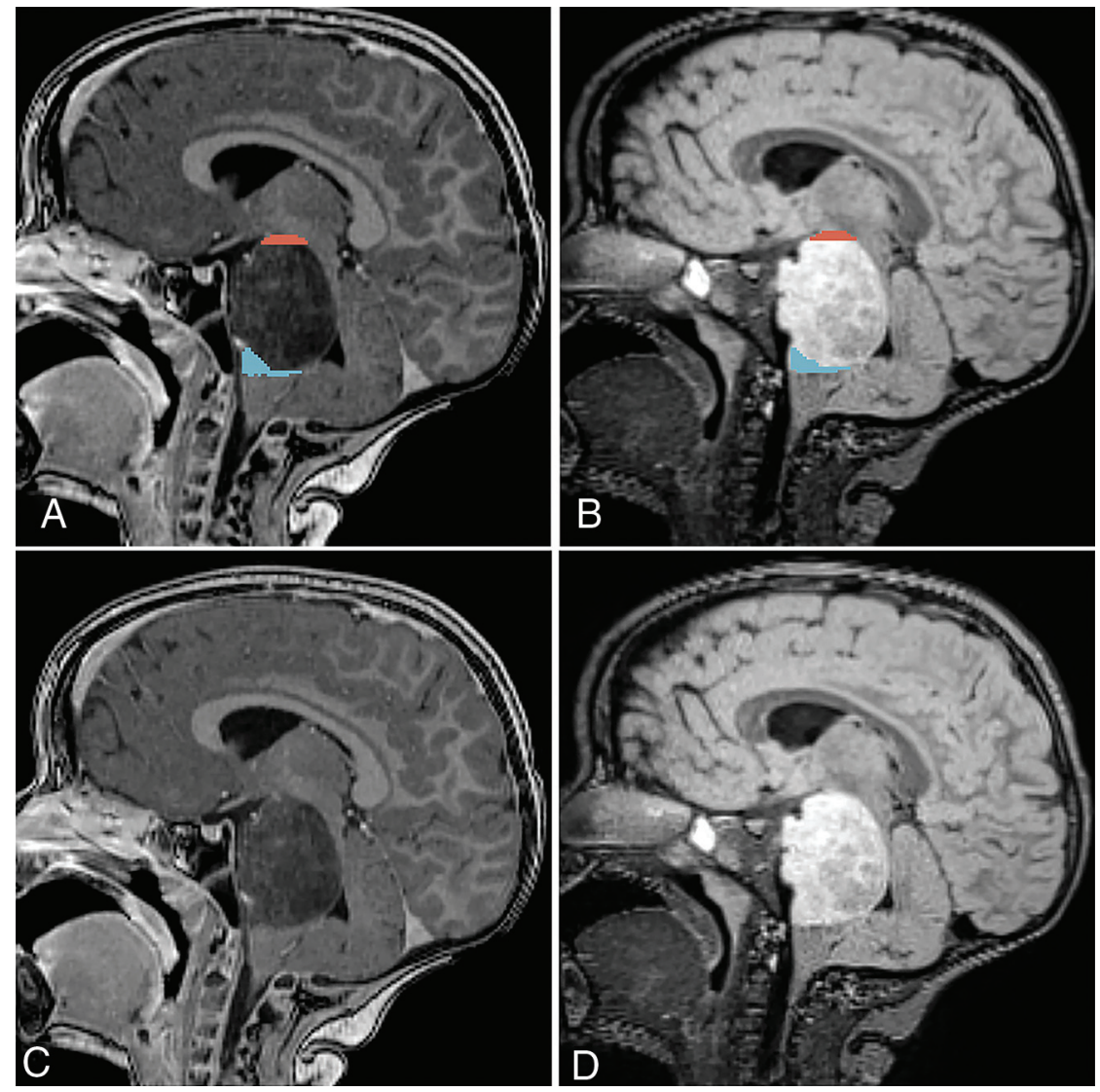

FIG 1. Vertical extrapontine lesion extensions in a patient with DIPG on sagittal postcontrast TIWI $(A$ and $C$ ) and T2WI FLAIR ( $B$ and $D$ ) scans. Segmented $(A$ and $B$ ) lesion extensions into the midbrain and medulla oblongata are highlighted in red and blue, respectively.

by a single, trained observer under the supervision of a board-certified neuroradiologist. Using the automatically detected TTV as a starting point, the TTV was adjusted in 3D Slicer (http://www.slicer.org) to correct any misclassification of image voxels. ${ }^{16}$ The median smoothing tool, with a 2to 3 -voxel radius, depending on the accuracy of each segment at the tumor borders, was used on individual segments during the adjustment process. ${ }^{17}$ Once the entire tumor was segmented, the PTV was isolated from the TTV using anatomic boundaries and landmarks. Any exophytism (typically ventral) that arose from the pons was included in the PTV. Often, the primarily intrapontine tumor had an exophytic component that prolapsed downward ventrally to the medulla, but a direct extension into the upper aspect of the medulla was also present. In these cases, sagittal and coronal views were used to distinguish the exophytic portion of the PTV and EPLE toward the medulla oblongata. Extrapontine tumor volume (ETV) was defined as the discordance between the PTV and the TTV. ETV was then further classified by location into extensions

was used for segmentation. Voxel size for the $3 \mathrm{D}$ acquisitions was isotropic $(1 \times 1 \times 1 \mathrm{~mm})$. The section thickness for $2 \mathrm{D}$ imaging sequences was consistently $4 \mathrm{~mm}$ without a gap; however, subsequently, all axial 2D images were interpolated to isotropic voxels $(1 \times 1 \times 1 \mathrm{~mm})$ and coregistered to the postcontrast $3 \mathrm{D} \mathrm{T} 1$ images.

The total tumor volume (TTV) was automatically estimated using a machine learning-based segmentation tool that we modeled after the work of Wang et al, ${ }^{15}$ which uses anisotropic convolutional neural networks to segment the entire tumor on the basis of precontrast T1-weighted and T2-weighted and postcontrast T1-weighted and FLAIR images. Postcontrast T2-weighted images were used for 2 subjects for whom precontrast $\mathrm{T} 2$-weighted images were unavailable. Subsequently, the images (including the TTV) were normalized to the Montreal Neurological Institute brain atlas. In the Montreal Neurological Institute space, the pons was segmented using SPM 12 software (http://www.fil. ion.ucl.ac.uk/spm/software/spm12); then, the resultant intrapontine tumor volume (pontine tumor volume [PTV]) was returned to the patient's space.

\section{Radiologic Variables}

After the automated segmentation of the tumors, each set of images and segmentations was evaluated and processed manually of the midbrain, medulla oblongata (collectively vertical EPLE), and right and left middle cerebellar peduncles (MCPs, collectively horizontal EPLE) (Figs 1 and 2). In addition to TTV, the craniocaudal dimension of the tumor was extracted from the segmented volume by subtracting the location of the most inferior section that contained the TTV from the most superior one. The presence of postcontrast enhancement was recorded for each extension as well as the pontine component of each tumor. The presence of ring enhancement was also recorded for each tumor.

\section{Statistical Analysis}

Descriptive statistics were calculated for variables of interest. Kaplan-Meier estimates were calculated to estimate the 1-, 2and 5-year OS and PFS rates and respective 95\% confidence intervals. The association between possible prognostic factors and OS data was explored by performing univariate linear regression because the data were not censored. Cox regression models were used to model the relationship between PFS and possible prognostic factors. Two-sample $t$ tests were used to assess whether imaging parameters were different between age categories ( 3 years or older at diagnosis versus younger than 3 years at diagnosis, age groups commonly used by other investigators). Patients were classified into 3 survival groups by their survival status (OS $>2$ years, OS between 1 and 2 years, and $\mathrm{OS}<1$ year). Continuous variables of ETVs were 

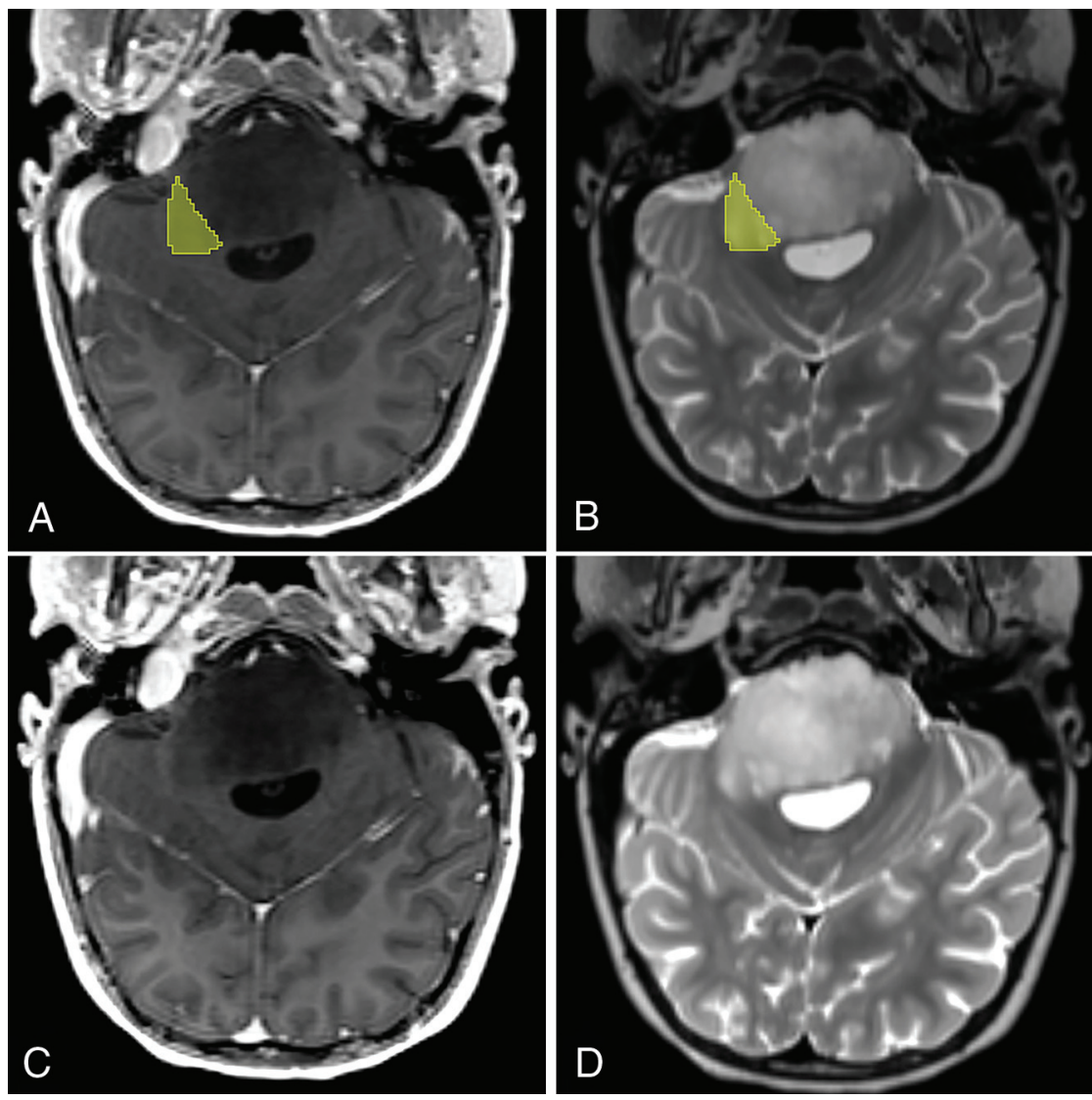

FIG 2. Unilateral horizontal extrapontine lesion extension in a patient with DIPG in transverse postcontrast TIWI ( $A$ and $C$ ) and T2WI $(B$ and $D)$. The segmented $(A$ and $B)$ extrapontine lesion extension toward the right middle cerebellar peduncle is highlighted in yellow. of metastasis and ETV), we used the Fisher exact test because there are cells in frequency tables with expected counts of $<5$. ANOVA was applied when evaluating the association between continuous variables (TLV and ETV) and categoric variables (pattern of metastasis, ring enhancement, and enhancement). A 2-sample $t$ test was performed to test the association between dichotomous enhancement pattern-related variables and continuous variables (global TLV/aggregate volume). All analyses were evaluated using SAS, Version 9.4 (SAS Institute, Cary, North Carolina).

\section{RESULTS}

\section{Survival Outcomes}

Of the 131 patients meeting the inclusion criteria, the median age at diagnosis was 6.4 years (interquartile range, 4.6 to 8.2). Ninety-eight $(74.8 \%)$ patients were white; 18 (13.8\%), black; demographics of the remaining 15 (12\%) are shown in Table 1. Seventy-eight (59.6\%) patients were female and $53(40.4 \%)$ were male, resulting in a female-to-male sex ratio of 1.5:1. The median PFS time was 6.8 months (interquartile range, 5.8 to 9.1 ). The respective 1-, 2-, and 5-year PFS rates were $16.20 \%(95 \% \mathrm{CI}, 10.44 \%-$

\section{Table 1: Patient characteristics}

\begin{tabular}{lc}
\hline \multicolumn{1}{c}{ Variable/Category } & No. (\%) \\
\hline Age at diagnosis & \\
3yr or older & $120(91.6)$ \\
Younger than 3yr & $11(8.4)$ \\
Sex & \\
$\quad$ Female & $78(59.6)$ \\
Male & $53(40.4)$ \\
Ethnicity & \\
Asian & $2(1.5)$ \\
Asian and white & $1(0.8)$ \\
Black & $18(13.8)$ \\
Black and white & $3(2.3)$ \\
Multiple & $5(3.8)$ \\
Other & $2(1.5)$ \\
Unknown & $2(1.5)$ \\
White & $98(74.8)$ \\
Metastasis at the end of progression-free survival? & \\
Yes & $24(18.3)$ \\
Spinal & $7(5.3)$ \\
Intracranial & $4(3.1)$ \\
Intracranial + spinal & $13(9.9)$ \\
No & $112(85.5)$ \\
\hline
\end{tabular}

compared among survival groups by performing ANOVA, and categoric variables of extrapontine extension were compared by performing $\chi^{2}$ testing. To evaluate the association between categoric variables (pattern of metastasis and EPLE pattern or pattern
23.09\%), 3.44\% (95\% CI, 1.14\%-7.90\%), and 0.86\% (95\% CI, 0.08\%$4.25 \%)$. At the time of progression, metastatic disease had developed in 24 patients (18.3\%), 7 with intracranial metastasis, 4 with spinal metastasis, and 13 with both spinal and intracranial metastasis. The presence of metastasis at progression was significantly associated with shorter PFS $(P=.01)$, but not with OS $(P=.09)$, pattern of extension $(P=.89)$, TTV $(P=.95)$, or ETV $(P=.39)$. Patient characteristics are summarized in Table 1 . The median OS time was 10.3 months (interquartile range, $7.4-14.9$ months). The respective 1-, 2-, and 5-year OS rates were 38.20\% (95\% CI, 29.89\%-46.39\%), $8.40 \%$ (95\% CI, $4.45 \%-13.92 \%$ ), and $0.76 \%$ (95\% CI, $0.07 \%-$ $3.82 \%)$. Kaplan-Meier curve OS rates of the cohort are shown in Fig 3. Analysis showed no difference in survival between age groups $(P=.65)$.

\section{Extrapontine Lesion Extension}

Baseline tumor volumes are summarized in Table 2. On the initial diagnostic MR imaging, 95.4\% $(n=125)$ of the tumors had at least 1 EPLE. The mean TTV, PTV, and ETV were $40.0279 \mathrm{~cm}^{3}$ (range, $8.9750-110.8010 \mathrm{~cm}^{3}$ ), $36.1874 \mathrm{~cm}^{3}$ (range, $8.9750-100.8550 \mathrm{~cm}^{3}$ ), and $4.03311 \mathrm{~cm}^{3}$ (range, $0.2840-16.9780 \mathrm{~cm}^{3}$ ), respectively (Table 2 ). The most common extension of EPLE was into the midbrain $83.2 \%(n=109)$, and the least common extension of EPLE, though still present in $61.8 \%$ of tumors, was into the MCPs $(n=81)$. Only $5.3 \%(n=7)$ of tumors had 


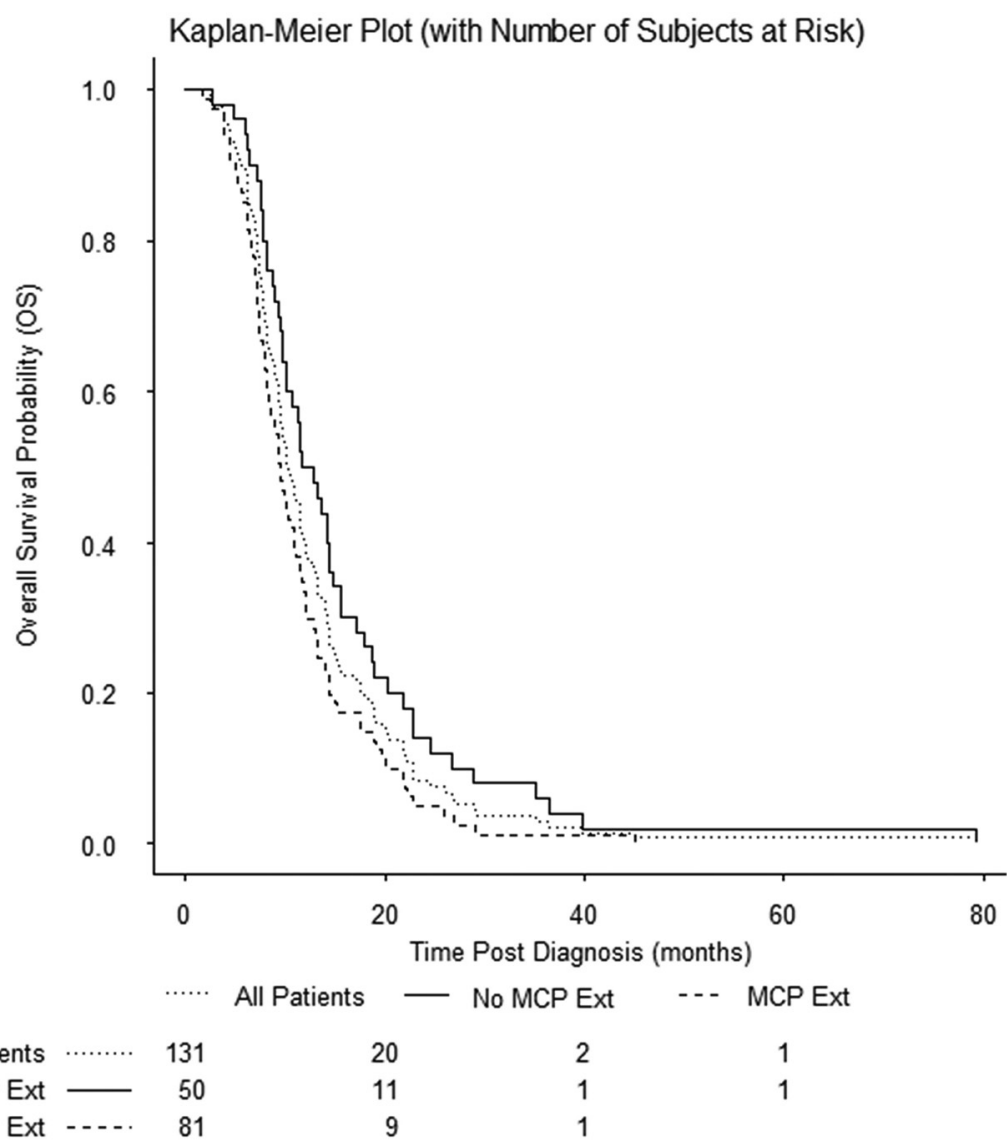

FIG 3. Kaplan-Meier plot of OS of the cohort. Any extension (Ext) into the middle cerebellar peduncles was prognostic of shorter overall survival $(P=.01)$.

Table 2: Tumor volumes at baseline

\begin{tabular}{lccc}
\hline \multicolumn{1}{c}{ VOI } & No. (\%) & Mean Volume $\left(\mathrm{cm}^{3}\right)$ (Range) & No. Enhancing (\%) \\
\hline TTV & $131(100.0)$ & $40.0279(8.9750-110.8010)$ & $106(80.92)$ \\
PTV & $131(100.0)$ & $36.1874(8.9750-100.8550)$ & \\
ETV & $125(95.4)$ & $4.03311(0.2840-16.9780)$ & $42(33.6)$ \\
EPLE, medulla oblongata & $101(77.1)$ & $0.96112(0.1910-4.5400)$ & $9(8.91)$ \\
EPLE, right MCP & $44(33.6)$ & $1.9368(0.0410-7.4560)$ & $14(31.11)$ \\
EPLE, left MCP & $44(33.6)$ & $1.7811(0.0750-7.8440)$ & $6(11.54)$ \\
EPLE, any MCP & $81(61.8)$ & $2.0196(0.0410-7.8440)$ & $18(22.22)$ \\
EPLE, midbrain & $109(83.2)$ & $2.23375(0.1380-11.9460)$ & $23(21.50)$ \\
\hline
\end{tabular}

\section{Postcontrast Enhancement}

At diagnosis, $80.92 \%$ of tumors ( $n=106)$ had some degree of postcontrast enhancement. Of those with enhancement, $50.9 \% \quad(n=54)$ were ring-enhancing lesions. A number of tumors $(22.1 \%, n=29)$ also showed occult enhancement, which we have previously identified as a potential marker of angiogenesis. ${ }^{7}$ Of 125 tumors with EPLE, $33.6 \% \quad(n=42)$ showed enhancement in at least 1 EPLE. The presence of postcontrast enhancement in individual EPLEs is summarized in Table 3. Enhancement of individual EPLEs was not associated with OS, PFS, an EPLE pattern, or TTV. The presence of any postcontrast enhancement at diagnosis was associated with increased ETV $(P=.02)$ and shorter OS $(P=.01)$, and enhancement of an EPLE into the midbrain was associated with increased ETV $(P=.01)$. The presence of ring enhancement was also associated with increased ETV $(P=.01)$. The presence of an MCP extension was not associated with the presence of enhancement $(P=.36)$, ring enhancement $(P=.82)$, or metastatic disease at progression $(P=1.00)$.

\section{DISCUSSION}

Our study, capitalizing on the largest single-center patient cohort to date, demonstrates that EPLEs are very common in DIPG and that their identification and quantitative analysis are feasible and, potentially, worthwhile in the clinical setting. Semiautomated segmentation enabled us to efficiently determine the frequency of the individual forms of EPLE and the various both right and left MCP extensions. Of the 16 potential combination patterns ( $>1$ EPLE associated with a tumor), 11 were present in the study population as shown in Fig 4. Most interesting, no horizontal EPLE was present without the concurrent presence of at least 1 vertical EPLE, either into the medulla oblongata or the midbrain.

The presence of any MCP extension was associated with shorter survival $(P=.01)$ on univariate analysis, but the volume of EPLE into the MCPs was not significant as a predictor of poorer OS $(P=.07)$. When we compared patterns of EPLE, patients with tumors with both vertical EPLEs without horizontal EPLE experienced significantly longer survival than did patients with any other pattern of EPLE $(P<.001)$ or those without any EPLE $(P=.04)$. The TTV, ETV (overall EPLE burden), and PTV were not significantly associated with OS. patterns defined by their possible combinations. We then found statistically significant correlations between horizontal EPLE (toward the MCPs) and shorter OS and vertical EPLE and longer survival, findings that have not been reported previously. We, therefore, submit that vertical and horizontal EPLEs should be evaluated separately because their effects on survival appear to have different, possibly opposing influences. Beyond their prognostic value, these findings may have implications for the targeting and dosing strategies used in fractionated conformal radiation therapy (RT), which provide only temporary relief but are the cornerstone of current treatment for DIPG. We think that these findings are meaningful additions to the existing body of the clinical and imaging knowledge base in DIPG.

The current overhaul of the definition of DIPG, including the poorly understood clinical implications, represents a limitation of 


\section{Patterns of Extrapontine Extension}

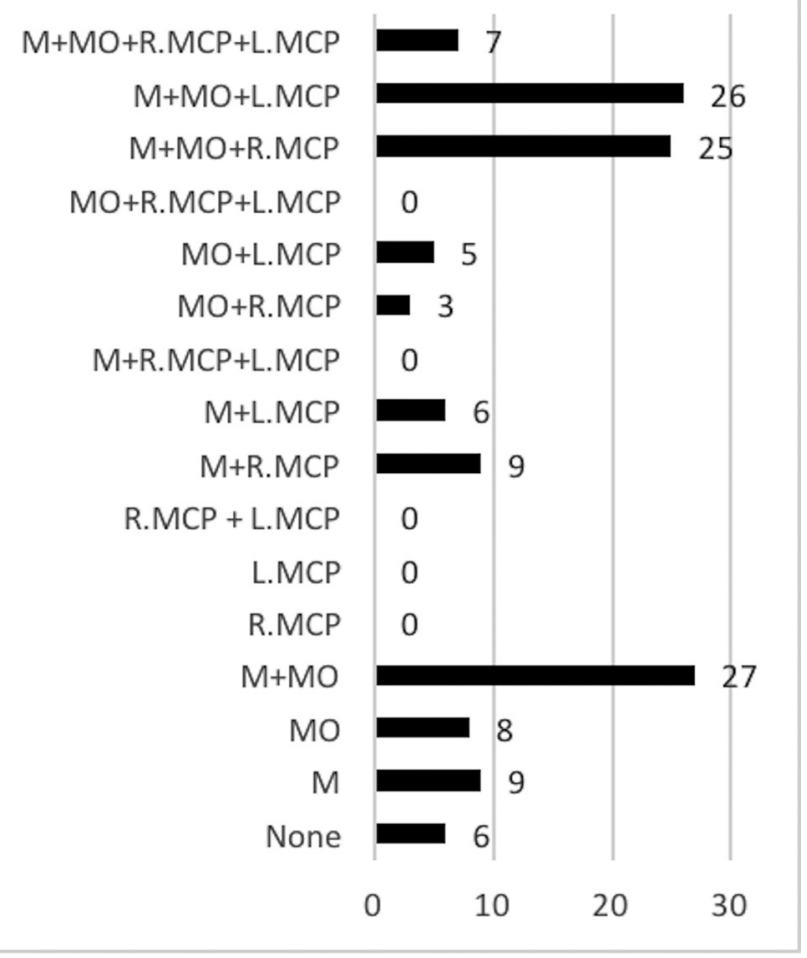

FIG 4. Frequency of patterns of extrapontine lesion extension. $M$ indicates midbrain; $M O$, medulla oblongata; L.MCP indicates left middle cerebellar peduncle; R.MCP, right middle cerebellar peduncle.

Table 3: Postcontrast enhancement in individual EPLEs

\begin{tabular}{lc}
\hline \multicolumn{1}{c}{ VOI } & No. Enhancing (\%) \\
\hline EPLE, medulla oblongata & $9(8.91)$ \\
EPLE, right MCP & $14(31.11)$ \\
EPLE, left MCP & $6(11.54)$ \\
EPLE, any MCP & $18(22.22)$ \\
EPLE, midbrain & $23(21.50)$ \\
\hline
\end{tabular}

our study. Because our study was retrospective and based on historical data, we used the now-obsolete term DIPG. This is, however, true for all previous similar studies, and we anticipate that large-scale prospective studies using the current, integrated approach introduced in the latest World Health Organization classification may not be available for several years. Another limitation of our study stems from the evolution of treatment regimens explored in our patients during the 17 -year review period. ${ }^{12,13}$ While the course of treatment was not analyzed in this study, changing protocols and the use of different chemotherapeutic or other tumor-targeted agents across the cohort should be considered. We found no significant difference in OS between patients treated in the 2 largest trials included in the cohort $(P=.480)$. After all, treatment heterogeniety is inevitable, given that no effective treatments for DIPG beyond RT have been identified and that new trials using novel agents must be constantly developed.

Approximately $80 \%$ of tumors radiographically diagnosed as DIPG in the past are thought to correspond to diffuse midline glioma H3 K27M-mutant neoplasms. Potentially clinically relevant, differences among $H 3$ wild-type, H3.1-, and H3.3-mutant tumors have been recognized recently in several reports. ${ }^{2,18}$ Unfortunately, the imaging and some of the biologic correlates of these tumor subgroups are still poorly understood because no consistent imaging features have been found to identify H3 K27M-mutant high-grade gliomas or to distinguish those from $\mathrm{H} 3$ wild-type tumors within the pons or elsewhere. This distinction depends on the availability of a biopsy at diagnosis or retrospectively at the time of postmortem examination; despite the recent advances in our understanding of the molecular and epigenetic underpinnings of DIPG (and other pediatric high-grade gliomas), the diagnosis of pediatric brain stem malignancies continues to rely heavily on imaging because of the persistently low rates of tissue biopsy at presentation $(<15 \%)$ in most countries, including the United States.

The lack of biopsy in our cohort represents a limitation of our study too. However, recent reports demonstrating the feasibility and accuracy of detecting $\mathrm{H} 3$ mutations in circulating tumor DNA within biofluids in these patients hold promise for minimally invasive diagnostic approaches in the future. ${ }^{19,20}$ In the meantime, the devastating nature of DIPG, and the lack of therapeutic advances necessitate the continued search for robust imaging biomarkers in pediatric DIPG/ DMG in the pursuit of enhanced prognostication and risk stratification at the time of diagnosis.

In prior studies, the methods for evaluating tumor volume have been inconsistent, while the description of the various EPLE patterns and the quantitative determination of the overall EPLE burden have been lacking. It is, therefore, difficult to estimate the contribution of EPLE to the overall lesion volume and its potential prognostic impact in those studies because the various forms and patterns of EPLE are not explicitly specified. ${ }^{5}$

In contrast, the strengths of our study include consistent imaging protocols across various patient subcohorts, a robust and highly automated imaging data analysis and processing pipeline, and the systematic use of tumor volume quantification instead of the descriptive approach used in prior studies. We also report demographic, expanded survival, and other data that have been sparsely available (eg, the frequency of leptomeningeal metastatic spread at the end of PFS). We report specific patterns of metastasis intracranially, intraspinally, or both and their prevalence in this cohort as well as a significant relationship between the presence of metastatic disease in the brain and spinal cord at progression with shorter PFS $(P=.01)$. The significance of this relationship is difficult to determine because the presence of metastatic disease was a criterion for progression in this cohort.

We have quantified the presence of postcontrast lesion enhancement in this cohort and identified relastionships between the presence of any enhancement at diagnosis and increased TLV and ETV as well as between enhancement at diagnosis and poorer OS.

Our research confirms that EPLE into the midbrain, the medulla oblongata, and the MCPs is common in DIPG and suggests 
that differences in survival outcomes exist among various patterns of EPLE. We propose 2 specific patterns of EPLE as predictors of OS in newly diagnosed DIPG; thus, our findings may be valuable for risk stratification in future clinical trials for children with newly diagnosed DIPG.

The findings of shorter survival in patients with any horizontal extension into the cerebellar peduncles and an apparent survival benefit in patients with craniocaudal EPLE without horizontal EPLE may have ramifications for RT targeting and treatment planning. It is possible that only the presence or absence of horizontal EPLE is the prognostically determinant factor. On the basis of limited postmortem examinations and patterns-of-failure studies, focal irradiation rather than wholebrain RT has been most commonly recommended for patients with DIPG. ${ }^{21-23}$ In the era of 3D conformal RT, clinical target volumes have ranged between 1 and $2 \mathrm{~cm}$ and have been expanded in an isotropic fashion. ${ }^{13,23}$ Although further work is needed to evaluate the patterns of failure and routes of spread after conformal RT, findings from this study suggest that a potential avenue of treatment intensification could include a "boost" dose RT to horizontal tumor extensions. Additionally, these data provide the opportunity to examine the extent of EPLE across these 3 principal directions of spread at diagnosis, with the goal of identifying rational nonisotropic clinical target volume margins.

\section{CONCLUSIONS}

EPLE in newly diagnosed DIPG is common. In a large cohort of patients with newly diagnosed DIPGs, we determined that the presence of EPLE into either MCP is predictive of shorter OS. We conclude that the identification and quantitiative analysis of EPLEs in DIPG are feasible, with potential clinical uses in the prognostication of DIPG at diagnosis and in the targeting and dosing strategies used in fractionated conformal RT.

Disclosures: Lydia Makepeace-RELATED: Grant: National Institutes of Health, National Cancer Institute, Comments: National Institutes of Health Cancer Center Support Grant P30 CA21765, Pediatric Oncology Education grant paid to me; National Cancer Institute grant R25CA23944, institutional grant.* Christopher L. Tinkle-UNRELATED: Payment for Manuscript Preparation: UpToDate, ependymoma chapter. Scott Hwang-UNRELATED: Employment: I am employed as a neuroradiologist; Grants/Grants Pending: National Institutes of Health, Comments: Five percent of my effort is supported by a grant for sickle cell anemia; $9 \%$ of my effort is supported by a grant for looking at sleep apnea in Hodgkin lymphoma survivors who received radiation therapy*; Stock/ Stock Options: I have stocks. None of these has any relationship to the research in the article; Travel/Accommodations/Meeting Expenses Unrelated to Activities Listed: My employer provides a small fund for professional development, which includes travel and room and board for Continuing Medical Education and other academic conferences. None was used for this article. Zoltan Patay-RELATED: Grant: National Institutes of Health, ALSAC, Comments: This work was supported by the National Institutes of Health Cancer Center Support Grant P30 CA21765, the National Cancer Institute grant R25CA23944, and ALSAC*; UNRELATED: Consultancy: Guerbet, Comments: Guerbet Medical Advisory Meeting, Chicago, July 17, 2016, honorarium; Travel/ Accommodations/Meeting Expenses Unrelated to Activities Listed: National Hospital for Neurology and Neurosurgery, University College, London, UK; Radiological Society of Sao Paulo; Hungarian Society of Neuroradiology; Turkish Society of Neuroradiology; European Congress of Magnetic Resonance in Neuropediatrics; European Society of Neuroradiology, Comments: visiting professorship, National Hospital for Neurology and Neurosurgery, University College, London, UK; invited speaker, Radiological Society of Sao Paulo, Brazil; invited speaker, Hungarian Society of Neuroradiology; invited speaker, Turkish Society of Neuroradiology; invited speaker, European Congress of Magnetic Resonance in Neuropediatrics; invited speaker, European Society of Neuroradiology. *Money paid to the institution.

\section{REFERENCES}

1. Dang M, Phillips PC. Pediatric brain tumors. Continuum (Minneap Minn) 2017;23:1727-57 CrossRef Medline

2. Hoffman LM, Veldhuijzen van Zanten SE, Colditz N, et al. Clinical, radiologic, pathologic, and molecular characteristics of long-term survivors of diffuse intrinsic pontine glioma (DIPG): a collaborative report from the International and European Society for Pediatric Oncology DIPG Registries. J Clin Oncol 2018;36:1963-72 CrossRef Medline

3. Tisnado J, Young R, Peck KK, et al. Conventional and Advanced Imaging of Diffuse Intrinsic Pontine Glioma. J Child Neurology 2016;31:1386-93

4. Barkovich AJK, Kun J, Packer LE, et al. Brain stem gliomas: a classification system based on magnetic resonance imaging. Pediatr Neurosurg 1990-1991;16:73-83 CrossRef Medline

5. Hargrave D, Chuang N, Bouffet E. Conventional MRI cannot predict survival in childhood diffuse intrinsic pontine glioma. $J$ Neurooncol 2008;86:313-19 CrossRef Medline

6. Jackson S, Patay Z, Howarth R, et al. Clinico-radiologic characteristics of long-term survivors of diffuse intrinsic pontine glioma. $J$ Neurooncol 2013;114:339-44 CrossRef Medline

7. Conway AE, Reddick WE, Li Y, et al. "Occult" post-contrast signal enhancement in pediatric diffuse intrinsic pontine glioma is the MRI marker of angiogenesis? Neuroradiology 2014; 56:405-12 CrossRef Medline

8. Jansen MH, Veldhuijzen van Zanten SE, Sanchez Aliaga E, et al. Survival prediction model of children with diffuse intrinsic pontine glioma based on clinical and radiological criteria. Neuro Oncol 2015;17:160-66 CrossRef Medline

9. Lober RM, Cho YJ, Tang Y, et al. Diffusion-weighted MRI derived apparent diffusion coefficient identifies prognostically distinct subgroups of pediatric diffuse intrinsic pontine glioma. J Neurooncol 2014;117:175-82 CrossRef Medline

10. Poussaint TY, Vajapeyam S, Ricci KI, et al. Apparent diffusion coefficient histogram metrics correlate with survival in diffuse intrinsic pontine glioma: a report from the Pediatric Brain Tumor Consortium. Neuro Oncol 2016;18:725-34 CrossRef Medline

11. Poussaint TY, Kocak M, Vajapeyam S, et al. MRI as a central component of clinical trials analysis in brainstem glioma: a report from the Pediatric Brain Tumor Consortium (PBTC). Neuro Oncol 2011;13:417-27 CrossRef Medline

12. Broniscer A, Baker JN, Tagen M, et al. Phase I study of vandetanib during and after radiotherapy in children with diffuse intrinsic pontine glioma. J Clin Oncol 2010;28:4762-68 CrossRef Medline

13. Broniscer A, Baker SD, Wetmore C, et al. Phase I trial, pharmacokinetics, and pharmacodynamics of vandetanib and dasatinib in children with newly diagnosed diffuse intrinsic pontine glioma. Clin Cancer Res 2013;19:3050-58 CrossRef Medline

14. Wetmore C, Turner D, Kun L, et al. PDCT-10: First in Pediatrics Phase 1 Study of Crenolanib Besylate (CP-868, 596-26) Administered During and After Radiation Therapy (RT) in Newly-Diagnosed Diffuse Intrinsic Pontine Glioma (DIPG) and Recurrent High Grade Glioma (HGG). Neuro Oncol 2016;18:vi147-48

15. Wang G, Li W, Ourselin S, Vercauteren T. Automatic brain tumor segmentation using convolutional neural networks with test-time augmentation. In: Crimi A, Bakas S, Kuijf H, Keyvan F, Reyes M, van Walsum T. eds. Brainlesion: Glioma, Multiple Sclerosis, Stroke and Traumatic Brain Injuries. BrainLes 2018, LNCS, 11384. Cham: Springer, 2019;61-72

16. Fedorov A, Beichel R, Kalpathy-Cramer J, et al. 3D Slicer as an image computing platform for the Quantitative Imaging Network. Magn Reson Imaging 2012;30:1323-41 CrossRef Medline 
17. 3D Slicer. 2018. http://www.slicer.org. Version 4.4.0 (2016)

18. Castel D, Philippe C, Calmon R, et al. Histone H3F3A and HIST1H3B K27M mutations define two subgroups of diffuse intrinsic pontine gliomas with different prognosis and phenotypes. Acta Neuropathol 2015;130:815-27 CrossRef Medline

19. Aboian MS, Solomon DA, Felton E, et al. Imaging characteristics of pediatric diffuse midline gliomas with histone H3 K27M mutation. AJNR Am J Neuroradiol 2017;38:795-800 CrossRef Medline

20. Huang TY, Piunti A, Lulla RR, et al. Detection of histone H3 mutations in cerebrospinal fluid-derived tumor DNA from children with diffuse midline glioma. Acta Neuropathol Commun 2017;5:28 CrossRef Medline

21. Mantravadi RV, Phatak R, Bellur S, et al. Brain stem gliomas: an autopsy study of 25 cases. Cancer 1982;49:1294-96 CrossRef Medline

22. Halperin EC. Pediatric brain stem tumors: patterns of treatment failure and their implications for radiotherapy. Int J Radiat Oncol Biol Phys 1985;11:1293-98 CrossRef Medline

23. Haas-Kogan DA, Banerjee A, Kocak M, et al. Phase I trial of tipifarnib in children with newly diagnosed intrinsic diffuse brainstem glioma. Neuro Oncol 2008;10:341-47 CrossRef Medline 